\title{
MEASURING TECHNIQUE BASED ON MACHINE VISION
}

\author{
Cheng Lizhou ${ }^{1}$ \\ ${ }^{1}$ Shanghai University of Engineering Science, School of Mechnical Engineering, Shanghai 201620, China
}

\begin{abstract}
Mechanical vision inspection technology is a new technology that integrates sensor, image processing and measurement technology. Mainly introducing the present situation and tendency of the development of machine vision, this paper expounds the basic concept of a typical mechanical vision inspection system and its important component, including camera, the lighting system, image acquisition card, image processing and output system, and introduces some algorithm that applied in the process of image processing. Finally, this paper introduces the application of the mechanical vision inspection system and the development trend of the mechanical vision inspection technology in the future.
\end{abstract}

Keywords: - Machine Vision; Inspection Technology; Image Processing; Measurement; Algorithm

\section{INTRODUCTION}

As an important part of human perception system, vision is the most important way for people to obtain external information, and the information provided by vision and the intuitive effect achieved by it are unmatched by other methods. Machine vision is a new technology that uses machines to replace and simulate the visual perception function of human eyes, and uses this function to realize measurement and detection. The application of machine vision for detection can not only greatly improve the detection efficiency, but also improve the detection accuracy. In addition, the machine vision detection technology belongs to automatic non-contact measurement. Compared with manual detection, it can carry out continuous high-precision measurement in large quantities.

\section{DEVELOPMENT STATUS AND TREND}

With the continuous development of computer technology, hardware and software technology, the detection system combined with software and hardware can often achieve a high degree of automatic measurement, thus attaining a high production efficiency. Meanwhile, unmanned, intelligent and highly automated production is increasingly becoming the mainstream, so, the detection system based on machine vision will become the development trend for a long time in the future.

Since the 12th five-year plan, the manufacturing of high-end intelligent equipment or high-end equipment has received great attention, and the development of machine vision and other discrete sensors, switching volume instruments and factory automation systems has been developed. Machine Vision technology has been proposed for more than 30 years and has already formed a branch of the industrial automation industry in the last 10 years[1].

"The serious situation of global energy crisis and environmental pollution, which makes the concept of low- carbon economy has become more and more deeply rooted in the hearts of people. Improving the industrial structure in order to reduce waste and increasing efficiency is very important to the development of enterprise, and also, combined with rising labor costs and labor shortage encourages companies to increase the investment to automation technology, in order to improve production efficiency. Machine vision automation equipment can work tirelessly and repeatedly, and machine vision will play an important role in some dangerous working environments that are not suitable for manual operation or where artificial vision cannot meet the requirements[2].

At present, what the principle of detection system based on machine vision is that use image taking device (mainly include CCD and CMOS) for image acquisition, and send information captured by $\mathrm{CCD}$ or CMOS to computer terminal through image acquisition cards, and then obtain useful information after image processing and analysis. Finally the system can calculate and output the final result through the preset algorithm.

\section{TESTING SYSTEM FRAMEWORK STRUCTURE}

A typical detection system based on machine vision includes light source, lighting system, camera, lens, image acquisition card, image processing, analysis program, detection and judgment algorithm and output module.

Machine vision is a comprehensive technology involving image processing, lighting system, sensor device and computer software and hardware technology. In a complete machine vision detection system, many aspects of technology will be integrated, but the most important technologies are light source, lighting technology, optical lens, camera, image acquisition card and image processing and analysis technology. The following is a brief description about the above key parts. 


\subsection{Light Source Lighting System}

Whether the light source is chosen suitably or not and whether the lighting system design is reasonable or not will all directly determine the final inspection quality of the machine vision inspection system. If the selected light source is not appropriate and the lighting design is not reasonable, the image features collected by the system will be blurred, and the brightness of the image, especially the picture contrast, will not meet the requirements. In this way, during image processing, image segmentation cannot be effectively carried out, and edges cannot be accurately captured. Under the circumstances, the result must be very variable, so, the system is always unreasonable. In contrast, as a non-transparent body, the detection part is more suitable to use the way of back lighting. The method of backlighting is that the object is placed between the light source and the camera. It can obtain high contrast images and facilitate subsequent image processing[3].

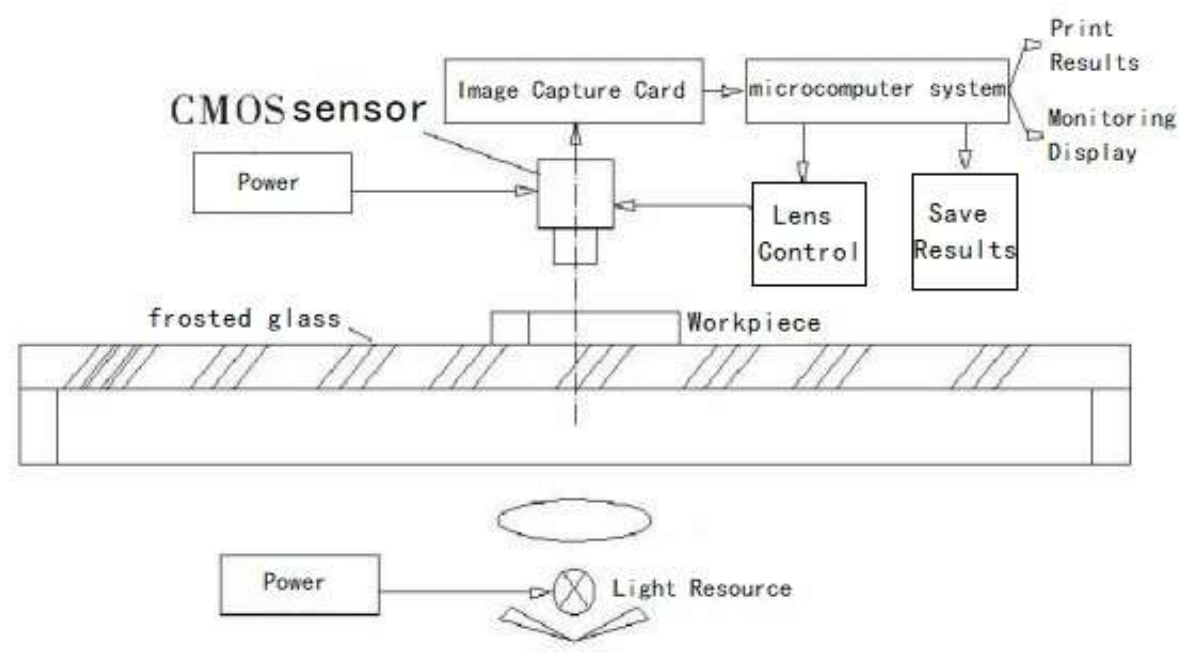

Fig 1:Schematic diagram of measurement system using backlighting method [4]

\subsection{Camera and Image Acquisition Card}

CCD image sensor collects information by collecting the optical signal of the workpiece under test and converting it into electrical signal, which is finally converted into digital signal through the circuit and transmitted to the image acquisition card. The CCD mainly consists of two structures, one is the wired array and the other is the plane array CCD.
The pixels in the structure of the CMOS image sensor are directly connected to ADC, which can directly convert the signal into digital signal, but will generate noise when transmitted over a long distance. However, CMOS also has the advantages of fast acquisition and processing speed, low power consumption and low price. The function of image acquisition card is to collect the acquired image information data into the computer in the digital form. Figure 2 shows the performance comparison between $\mathrm{CCD}$ and $\mathrm{CMOS}$ image sensors.

\begin{tabular}{ccc}
\hline Performance Parameters & CCD & CMOS \\
\hline sensitivity & E & $\mathrm{G}$ \\
noise & $\mathrm{E}$ & $\mathrm{G}$ \\
halo & with & without \\
power supply & multielectrode & single electrode \\
intergration & low & high \\
system consumption & high & low \\
circuit structure & complicated & simple \\
anti-radiation & low & high \\
dynaminc range & $>70$ db & $>70 \mathrm{db}$ \\
module size & large & small \\
color coding & off-chip & on-chip \\
ADC module & off-chip & on-chip \\
timing and control circuits & off-chip & on-chip \\
automatic gain control & off-chip & on-chip \\
\hline
\end{tabular}

Fig 2:Performance comparison between CCD and CMOS image sensor[5] 


\subsection{Image Processing Software}

At present, the most widely used software in industry is $\mathrm{VC}++$ series. With industrial cameras, the manufacturer has provided corresponding development packages, such as OpenGL and OpenCV. There is also a combination of software based on Labview and Matlab. Labview has powerful functions in data collection, measurement and control, and its own visual development module is convenient for users to use, while Matlab has powerful functions in image processing.

\section{VISUAL IMAGE PROCESSING}

After collecting the image information of the workpiece under test through camera, the computer image processing algorithm directly determines the final measurement accuracy. The whole software processing part mainly includes system calibration, image preprocessing and result calculation.

\subsection{System Calibration}

In the machine vision detection system, in order to use the obtained $2 \mathrm{~d}$ image for detection, it is necessary to match the $2 \mathrm{~d}$ image with the $3 \mathrm{~d}$ entity and establish the deterministic relationship between the two, that is, the camera's imaging model. According to the relationship between the two, the obtained image can be transformed into measurement data.

The purpose of camera calibration is to use the reference point coordinates of a given object $(\mathrm{x}, \mathrm{y}, \mathrm{z})$ and its image coordinates $(\mathrm{u}, \mathrm{v})$ to calculate the geometric and optical properties (internal parameters) within the camera and the coordinates of the camera in the three-dimensional world (external parameters). Internal parameters include focal length $\mathrm{f}$, lens distortion coefficient $(\mathrm{k}, \mathrm{s}, \mathrm{p})$, coordinate distortion factor $\mathrm{s}$, image coordinate origin $(\mathrm{u} 0, \mathrm{v} 0)$ and other parameters. External parameters include the rotation matrix $\mathrm{R}$ and the displacement vector $\mathrm{T}$ of the camera coordinate system relative to the world coordinate system[6].

Generally speaking, there are two methods for camera calibration. One is to use objects of known size as reference objects, and then obtains the parameters of camera imaging model through image processing and a series of transformations and calculations. We call this calibration method the traditional calibration method. The implement of traditional camera calibration method also determines its defects. The acquisition of parameters is overdependent on preset object, so, it is inconvenient because camera adjustment is often required in practice. Therefore, there is a calibration method that uses the internal parameters of the camera without relying on the external environment, which is called camera self-calibration.

At present, the traditional camera calibration method can be divided into the following three types according to its solution method: linear calibration method, nonlinear optimization calibration method and two-step calibration[7].
FIG. 3 shows the world coordinate system, image plane coordinate system, camera coordinate system and image physical coordinate system, which is needed in the process of camera imaging.

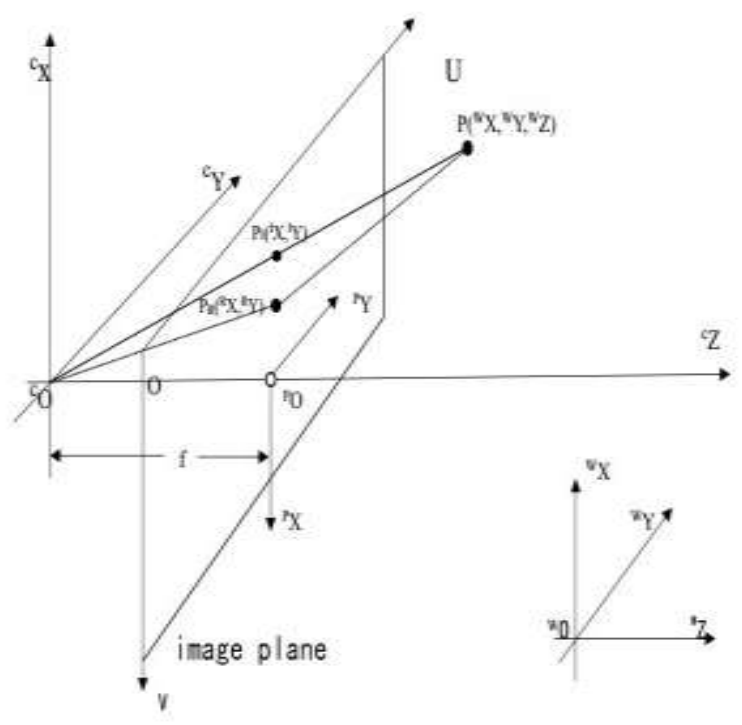

Fig 3: Coordinate relations in camera imaging

The world coordinate system is a customized threedimensional coordinate system. The image plane coordinate system is a rectangular plane coordinate system in pixels. The two axes correspond to the $\mathrm{X}$ and $\mathrm{Y}$ axes of the image.The camera coordinate system takes the camera's optical center as the origin of the coordinates, the direction along the optical axis as the $\mathrm{Z}$ axis, and $\mathrm{f}$ as the focal length of the camera.The image physical coordinate system is established at the intersection of the optical axis and the image plane, which is parallel to the camera coordinate axis.

\subsection{Image Preprocessing}

In the machine vision detection system, CCD or CMOS is used for image acquisition. Since it is color images that system collects, image preprocessing is required to facilitate edge detection and information extraction in the later stage. Image preprocessing mainly includes image grayscale, image filtering and enhancement, image binarization and image edge detection. The block diagram describes images processing flow is shown in figure 4. 


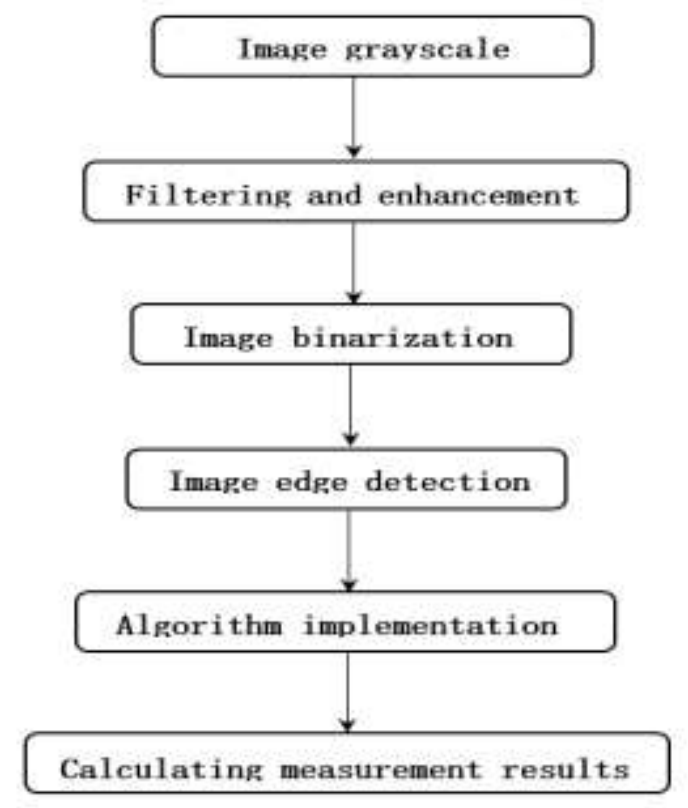

Fig 4: Block diagram of image processing flow

\subsubsection{Image Grayscale}

Color images are usually composed of three channels. In the corresponding processing process, in order to improve the processing speed, the image should be grayed, that is, a grayscale value is used to represent the values of $R, G$ and $B$ channels, so as to obtain the grayscale image of a single channel. Common methods of grayscale include single component method, maximum value method, average method and weighted average method. The grayscale image, which is reasonable and meets actual requirements, can be obtained by using weighted average method.

\subsubsection{Image Filtering and Enhancement}

In the process of image recognition, the images captured by the camera will be interfered by noises during the process of digitization and transmission, so the quality of the images will often decline. Usually, these noises will blur the images and edge features, which is not conducive to image processing and analysis. Therefore, it is very important to suppress the various interference signals that degrade the image, enhancing the useful signals in the image, and correct different observed images under the same constraint conditions. The filtering principle and method used in the pretreatment are described below.

\subsubsection{Gaussian Filtering}

Gaussian filtering is a kind of linear smoothing filtering, which can effectively filter the gaussian white noise in the image and has been widely used in the preprocessing stage of image processing. Gaussian filtering is the process of weighted average applied to the entire image, that is, the weighted average gray value of the pixel in the neighborhood is used as the value of the pixel point. After gaussian filtering, useful information can be retained.

\subsubsection{Mean Filtering}

Mean filtering is also called linear filtering. It mainly adopts neighborhood average method. Extracting several pixel points in the neighborhood, find the mean value, and assign the mean value to the current pixel point. Because the neighborhood average method is adopted, the noise is effectively suppressed, but at the same time, the defects brought by this method result in blurred image, damage the edge of the image and drown the edge features.

\subsubsection{Median Filtering}

Median filtering is a nonlinear smoothing technique in which the median gray value of all pixel points in a certain neighborhood is taken as the gray value of the pixel point. The median filter can suppress noise while protecting edge information, especially for pepper and salt noise.

\subsection{Image Binarization}

Before measuring the dimension of the workpiece, it is necessary to obtain accurate image information, which requires image binary processing. Image binarization is a kind of basic image processing technology, its core is to select one or multiple appropriate grey values to after image graying, this gray value is called the threshold. Dividing grayscale images with selected threshold, which can distinguish useful information from background, so as to obtain valuable image information.

The basic process of binarization is as follows: (1)Filtering the original image with medium and low pass, preprocessing the image, reducing or removing noise; (2) Parliamentary algorithm is used to determine the optimal threshold value; (3) The grayscale value of any pixel greater than this threshold is set to 255 , and less than this threshold is set to 0 . In this way, the processed image only has black and white colors, thus dividing the grayscale range into two categories: target and background, achieving image binarization[8]. The initial binarization method adopts the fixed threshold, which is simple to implement. However, for images with low contrast, information will be omitted, which is not conducive to image processing and analysis. After that, the fixed threshold binarization method is improved and the basic global threshold binarization method is generated. The basic processing flow is as follows: (1)The initial estimate parameter (such as the average grayscale of the image) is selected as $\mathrm{T}$; (2) Parliamentary $\mathrm{T}$ is used to segment images. This produces two sets of pixels: G1 consists of all pixels with a grayscale greater than T; G2 consists of all pixels with grayscale less than or equal to $\mathrm{T}$; (3) Calculate the average grayscale value of all pixels between $\mathrm{G} 1$ and G2. (4) To calculate a new threshold value: $T^{\prime}=1 / 2(\mu 1+\mu 2)$; (5)Terminate repeated iterative operation after difference between $\mathrm{T}^{\prime}$ and $\mathrm{T}$ is less than the pre-defined parameter T0[9].

Among global binarization methods, OTSU is regarded as the most classical algorithm, and OTSU is also called the 
maximum inter-class variance method, which is an efficient and fast algorithm to realize threshold self-adaptation. There is also a local binarization method, Bernsen algorithm is a exactly typical local binarization algorithm.

\subsection{Edge Detection}

The edges of images are often irregular or unstable, and there are often signal mutations. However, the edge features always contain a lot of information, so, it is necessary to detect the edge of the image during image processing. Most commonly, edge detection is defined as the process, which can describe grayscale changes in images accroding to the physical process of grayscale changes that causes grayscale changes of image. In the actual processing, the edge often contains a lot of noises, which requires image filtering and enhancement before edge detection. The noise should be removed as much as possible while retaining edge features, and the edge enhancement can also be appropriately carried out. As mentioned in literature [10], the edge is the place where the grayscale of the image changes dramatically. Therefore, in the earliest edge detection, the maximum value of the first derivative or the zero of the second derivative is used to detect the edge point. On this basis, different differential operators are derived, such as Sobel operator, Roberts operator, Prewitt operator and Laplacian operator. In addition, Canny edge detection operator, edge detection operator of fuzzy reasoning and Mallat wavelet edge detection operator are also mentioned in literature [11].

\subsubsection{First-Order Differential Method}

The gradient function of image symbolizes the rate of change about grayscale, which is the local maximum on the edge. Gradient operator or first derivative operator is used to estimate the direction of image grayscale change, enhancing the region of gray scale change in the image, and then furtherly judge the edge in the enhanced region.

For continuous function $I(x, y)$, its first order directional derivative in the direction, direction and direction of the point $(x, y)$ is:

$$
\begin{aligned}
& I_{x}(x, y)=\frac{\partial I(x, y)}{\partial x} \\
& I_{y}(x, y)=\frac{\partial I(x, y)}{\partial y} \\
& I_{\theta}(x, y)=\frac{\partial I(x, y)}{\partial x} \cos \theta+\frac{\partial I(x, y)}{\partial y} \sin \theta
\end{aligned}
$$

Define gradient at point $(x, y)$ as a vector:

$$
\nabla I(x, y)=\left[G_{x} G_{y}\right]^{T}=\left[\begin{array}{ll}
\frac{\partial I}{\partial x} & \frac{\partial I}{\partial y}
\end{array}\right]^{T}
$$

Gradient magnitude :

$$
|\overrightarrow{\operatorname{grad}}|=\sqrt{\left(\frac{\partial}{\partial x}\right)^{2}+\left(\frac{\partial}{\partial y}\right)^{2}}
$$

The gradient direction is perpendicular to the edge :

$$
\varphi=\tan ^{-1}\left(\frac{\partial}{\partial y} / \frac{\partial}{\partial x}\right)
$$

On the basis of this theory, different kinds of edge detection operators such as Sobel operator, Roberts operator, Prewitt operator and so on are derived.

\subsubsection{Second-Order Differential Method}

At the edge, the step of gray value will be produced, so the rate of change is maximum. The second derivative is solved by the method of zero crossing. In the direction of the point $(x, y)$, the second derivative of the direction $(x, y)$ is:

$$
\begin{aligned}
& I_{x x}(x, y)=\frac{\partial^{2} I(x, y)}{\partial x^{2}} \\
& I_{y y}(x, y)=\frac{\partial^{2} I(x, y)}{\partial y^{2}}
\end{aligned}
$$

The second derivative of $I(x, y)$ along the gradient direction at the point $(x, y)$ can be obtained as:

$\frac{\partial^{2} I(x, y)}{\partial \overrightarrow{n^{2}}}=\frac{\partial^{2} I(x, y)}{\partial x^{2}} \cos ^{2}(\theta)+2 \frac{\partial^{2} I(x, y)}{\partial x \partial y} \sin (\theta) \cos (\theta)+\frac{\partial^{2} I(x, y)}{\partial y^{2}} \sin ^{2}(\theta)$

Laplacian operator with linear and rotational symmetry is derived from the second-order derivative operator:

$$
\nabla^{2}=\frac{\partial^{2}}{\partial x^{2}}+\frac{\partial^{2}}{\partial y^{2}}
$$

The Sobel operator carries out edge detection according to the feature of gray scale weighting difference in pixel field, and reaches the extreme value at the edge. This method has a smoothing effect on noise. The edge positioning accuracy is not high enough. Roberts operator uses the difference between two pixels adjacent to the diagonal to approximate 
the gradient amplitude to detect the edge, with high positioning accuracy and sensitivity to noise.The Prewitt operator uses the gray difference between the top and bottom of pixel points, the left and right adjacent points to reach the extreme value at the edge to detect the edge, and the effect is similar to Sobel operator. Laplacian operator is a second-order differential operator, which uses zerocrossing of the second derivative at the edge point to detect the edge. It is sensitive to the change of gray value, has high positioning accuracy and is sensitive to noise, so it cannot obtain edge direction information. Canny is a relatively new detection operator, which can also play a certain role in noise suppression while realizing edge detection.

With the continuous development of edge detection technology, the algorithm should be able to accurately detect effective edges in edge detection, with high positioning accuracy, and should be insensitive to noise and less affected by edge direction. Therefore, in order to meet the precision requirement of image processing, modern advanced operators should be summarized on the basis of the existing traditional operators.

\section{DISCUSSION}

When making dimension measurement based on machine vision, we can collect information through camera and image acquisition card, and then use software such as Labview and Matlab for image processing. With the continuous improvement of industrial cameras, cameras with higher resolution and less distortion can be selected in the future, which can greatly improve the measurement accuracy.

At the same time, as the most critical part of the machine vision detection system, and also the most complex technical problem, the advantages and disadvantages of the algorithm directly affect the efficiency and quality of measurement, which can be improved on the basis of existing algorithms, so as to obtain high precision and high efficiency processing methods. As mentioned in the literature [12], in the process of actual operation, for industrial machine vision systems, for a certain scenario, a typical system mainly consists of following serval parts: image acquisition, image preprocessing, image segmentation, feature extraction, classification and inspection and final action, and the most critical among above serval parts are image acquisition, image processing and calculation of the system. In order to achieve the desired effect on the whole system, each of the described parts should be reasonably optimized.

\section{CONCLUSION}

In this paper, the hardware, software and algorithms of the dimension measurement system based on machine vision are introduced, and the principles, methods and current situation of these parts are explained in turn. A complete machine vision inspection system should include light source and lighting, optical lens and camera, image acquisition card and the core image processing technology. In particular, the filtering and enhancement in image preprocessing and the operators used in edge detection are described in detail.

With the continuous development of machine vision technology, machine vision technology plays an increasingly important role in industrial manufacturing. Besides dimension measurement, machine vision technology also participates in the monitoring and control of manufacturing process. In the literature [13], we can see that the combination of machine vision and control can make it more convenient to monitor various aspects of production products, and the combination with specific modules can also detect and evaluate the quality of products. In industrial production, extensive use of machine vision technology can accelerate the transformation of manufacturing industry to automatic manufacturing, which is also a trend of manufacturing industry development in recent years.

\section{REFERENCES}

[1] Bian Zheng-gang. The Development of Machine Vision Technology[J]. China Instrumentation, 2015,(06):40-42+65.

[2] Liu Yanqiu. The development of machine vision technology $[\mathrm{J}]$. Electronic Components and Materials,2014,(05):93-94.

[3] Qi Xiaoling, Zhao Xiaoxiao, JinWuyin. Geometric dimension measurement of shaft parts based on machine vision[J]. Modular Machine Tool \& Automatic Manufacturing Technique,2013,(01):65$67+73$.

[4] Liu Zhongpo, Du Baojiang, Zhang Jie. Automatic measuring system of part size based on machine vision[J]. Precise Manafacturing\& Automation,2006,(02):47-49.

[5] Wang Xudong, Ye Yvtang. The comparative research and development trend between CMOS and CCD image sensors $[\mathrm{J}]$. Electronic Design Engineering,2010,(11):178-181.

[6] Li Peng, Wang Jun-ning. Overview of Camera Calibration Methods[J]. Shanxi Electronic Technology,2007,(04):77-79.

[7] Li Mingming. Online measurement of parts based on computer vision inspection technology[J]. Laboratory Research and Exploration,2013,(05):105-107.

[8] Sun Shaolin, Ma Zhiqiang, Tang Wei. Study on gray image binarization algorithm[J]. Value Engineering,2010,(05):142-143.

[9] Jiang Ming, Liu Hui, Huang Huan. Research on image binarization technology $[\mathrm{J}]$. Software Guide,2009,(04):175-177.

[10] Dong Hongyan. Several technical studies on edge detection[D]. National University of Defense Technology,2008.

[11] Liu Zhongpo, Du Baojiang, Zhang Jie. Automatic measuring system of part size based on machine vision[J]. Precise Manafacturing\& Automation,2006,(02):47-49. 
[12] H.Golnabi, A.Asadpour. Design and application of industrial machine vision systems[J]. Robotics and Computer-Integrated Manufacturing ,2007(23):630637.

[13] Massimo Pacella, Antonio Grieco, MarziaBlaco. Machine vision based quality control of free-form profiles in automatic cutting processes[J]. Computers\&Industrial Engineering,2017(109):221 232. 